\title{
Using visuo-kinetic virtual reality to induce illusory spinal movement: The MoOVi Illusion
}

\author{
Daniel S Harvie Corresp., ${ }^{1}$ ， Ross T Smith ${ }^{2}$ ， Estin V Hunter ${ }^{1}$ ， Miles G Davis ${ }^{2}$ ， Michele Sterling ${ }^{1}$, G. Lorimer \\ Moseley ${ }^{3,4}$ \\ ${ }^{1}$ Recover Injury Research Centre, Centre of Research Excellence in Road Traffic Injury, Menzies Health Institute QLD, Griffith University, Griffith \\ University, Gold Coast, Australia \\ 2 Wearable Computer Lab, University of South Australia, Adelaide, Australia \\ ${ }^{3}$ Sansom Institute for Health Research, University of South Australia, Adelaide, Australia \\ ${ }^{4}$ Neuroscience Research Australia, Sydney, Australia \\ Corresponding Author: Daniel S Harvie \\ Email address: d.harvie@griffith.edu.au
}

\section{Abstract}

Background: Illusions that alter perception of the body provide novel opportunities to target brainbased contributions to problems such as persistent pain. One example of this, mirror therapy, uses vision to augment perceived movement of a painful limb to treat pain. Since mirrors can't be used to induce augmented neck or other spinal movement, we aimed to test whether such an illusion could be achieved using virtual reality, in advance of testing its potential therapeutic benefit. We hypothesised that perceived head rotation would depend on visually suggested movement.

Method: In a within-subjects repeated measures experiment, 24 healthy volunteers performed neck movements to $50^{\circ}$ of rotation, while a virtual reality system delivered corresponding visual feedback that was offset by a factor of $50 \%$ to $200 \%$ - the Motor Offset Visual Illusion (MoOVi) - thus simulating more or less movement than that actually occurring. At $50^{\circ}$ of real-world head rotation, participants pointed in the direction that they perceived they were facing. The discrepancy between actual and perceived direction was measured and compared between conditions. The impact of including multisensory (auditory and visual) feedback, the presence of a virtual body reference, and the use of $360^{\circ}$ immersive virtual reality with and without three-dimensional properties, was also investigated.

Results: Perception of head movement was dependent on visual-kinaesthetic feedback ( $p=0.001$, partial eta squared $=0.17$ ). That is, altered visual feedback caused a kinaesthetic drift in the direction of the visually suggested movement. The magnitude of the drift was not moderated by secondary variables such as the addition of illusory auditory feedback, the presence of a virtual body reference, or threedimensionality of the scene.

Discussion: Virtual reality can be used to augment perceived movement and body position, such that one can perform a small movement, yet perceive a large one. The MoOVi technique tested here has clear potential for assessment and therapy of people with spinal pain. 
1 Using visuo-kinetic virtual reality to induce illusory spinal movement: The MoOVi

2

3

4

5

6

7

8

9

10

11

12

13

14

15

16

17

18

19

20

21

22

23

24

25

26

27 Correspondence concerning this article should be addressed to Daniel Harvie, Griffith 28 29

30

31

32
Daniel S. Harvie ${ }^{1}$, Ross T. Smith ${ }^{2}$, Estin Hunter ${ }^{1}$, Miles Davis ${ }^{2}$, Michele Sterling ${ }^{1}$, G. Lorimer Moseley 3,4

${ }^{1}$ Recover Injury Research Centre, NHMRC CRE in Recovery Following Road Traffic Injury, Menzies Health Institute QLD, Griffith University, Gold Coast, Australia

${ }^{2}$ Wearable Computer Lab, University of South Australia

${ }^{3}$ Sansom Institute for Health Research, University of South Australia

${ }^{4}$ Neuroscience Research Australia, Randwick, Australia

\section{7}

8

9

0

University, Gold Coast Campus, Southport QLD 4222. E-mail: d.harvie@griffith.edu.au, T: +61 755529774, F: +61883022358. 


\section{Abstract}

Background: Illusions that alter perception of the body provide novel opportunities to target brain-based contributions to problems such as persistent pain. One example of this, mirror therapy, uses vision to augment perceived movement of a painful limb to treat pain. Since mirrors can't be used to induce augmented neck or other spinal movement, we aimed to test whether such an illusion could be achieved using virtual reality, in advance of testing its potential therapeutic benefit. We hypothesised that perceived head rotation would depend on visually suggested movement.

43 Method: In a within-subjects repeated measures experiment, 24 healthy volunteers performed neck movements to $50^{\circ}$ of rotation, while a virtual reality system delivered corresponding visual feedback that was offset by a factor of 50\% to $200 \%$ - the Motor Offset Visual Illusion (MoOVi) - thus simulating more or less movement than that actually occurring. At $50^{\circ}$ of real-world head rotation, participants pointed in the direction that they perceived they were facing. The discrepancy between actual and perceived direction was measured and compared between conditions. The impact of including multisensory (auditory and visual) feedback, the presence of a virtual body reference, and the use of $360^{\circ}$ immersive virtual reality with and without three dimensional properties, was also investigated.

Results: Perception of head movement was dependent on visual-kinaesthetic feedback ( $p=$ $0.001, \eta_{p}^{2}=0.17$ ). That is, altered visual feedback caused a kinaesthetic drift in the direction of the visually suggested movement. The magnitude of the drift was not moderated by secondary variables such as the addition of illusory auditory feedback, the presence of a virtual body reference, or three-dimensionality of the scene.

Discussion: Virtual reality can be used to augment perceived movement and body position, such that one can perform a small movement, yet perceive a large one. The MoOVi technique tested here has clear potential for assessment and therapy of people with spinal pain. 
64

65

66

67

68

69

\section{Introduction}

70 The relationship between the brain's representations of the body and the integrity of the body

71 itself is complex. In both recovery from an acute injury and in the transition from acute to

72 chronic pain, resolution of tissue pathology and resolution of pain do not go hand in hand. One

73 explanation for ongoing pain after tissue healing is that the brain retains neural encoding

74 consistent with a body that is still threatened - the brain misrepresents the body. This threatened 75 state then continues to drive redundant protective responses such as pain. Thus, chronic pain 76 could be viewed as a mismatch between the body and its neural representation. This theme is 77 common to several contemporary theories of chronic pain [(see cite for examples and review of 78 such theories - the cortical body matrix paradigm (Moseley, Gallace \& Spence 2012), the 79 Imprecision Hypothesis of chronic pain (Moseley \& Vlaeyen 2015), Explain Pain (Moseley \& 80 Butler 2015)].

81

Illusions that distort bodily perception confirm that divergence between real and perceived bodily state is possible, and that the brain's representation of the body can be artificially manipulated. The flagship example of this is the rubber hand illusion (RHI). To perform the RHI, the participant's hand is hidden, and a rubber hand is placed in view. The experimenter simultaneously strokes the out-of-view real hand, and the in-view rubber hand. Within a minute, most participants report feeling touch coming from the rubber hand, to which they also report a sense of agency (Botvinick and Cohen 1998). Further, when asked to point to their hand, a shift in perceived limb position towards the rubber hand is apparent: a kinaesthetic drift (Botvinick and Cohen 1998). This illusion is possible by the integration of seen and felt sensory inputs and engages a range of physiological and perceptual processes (Moseley et al. 2008; Barnsley et al.

92 2011; Moseley, Gallace \& Spence 2012). 
94 Mirror therapy is an effective treatment for phantom limb pain and may have application in other

95 types of pathological hand pain (Chan et al 2007; Cacchio et al. 2009; Ramachandran \&

96 Altschuler. 2009; Bowering et al. 2012). It appears to utilise a similar illusory principle to that

97 observed for the RHI. In mirror therapy, the subject places the affected limb behind a mirror and

98 observes the moving reflection of the healthy limb. The subject concurrently performs a partial

99 or imagined movement of the painful or missing limb. The integration of motor commands, with

100 proprioceptive and or visual information, results in an illusory perception of movement that

101 aligns with the movement of the mirrored hand. This augmented perception may be viewed as

102 one of a brain convinced that the affected limb is in a state similar to that of the unaffected

103 side — present, free moving, and healthy — which may counter neural encoding that continues to

104 hold the body as threatened.

105

106 When effective, illusions such as mirror therapy must act via brain-based mechanisms. Indeed,

107 pathological hand and phantom limb pain are associated with signs of central dysfunction,

108 including aberrant perception of size and shape of the body part, and functional changes in the

109 somatosensory cortex (Marinus et al. 2011). Evidence consistent with distorted cortical body

110 representation in chronic pain, however, is not unique to painful limbs. For example, sensory

111 changes and functional reorganization of the primary somatosensory cortex has been observed

112 not only in pathological hand pain (Juottonen et al. 2002; Di Pietro et al. 2013; Di Pietro et al.

113 2015), carpal tunnel syndrome (Tecchio et al. 2002), and phantom limb pain (Flor et al. 1995),

114 but also in spinal pain conditions (Flor et al. 1997; Catley et al. 2014). The extent of this

115 reorganization is associated with the characteristics of pain including intensity (Flor et al. 1995)

116 and duration (Flor et al. 1997), suggesting their relevance to clinical symptoms.

117

118 Given the potential contribution of the brain to ongoing spinal pain, cortical representations

119 could be a valuable target for intervention. This prospect is particularly relevant for a number of

120 reasons. Firstly, spinal pain — specifically neck and lower back pain — together form the single

121 greatest contribution to years lived with disability world-wide (Vos et al. 2015). Secondly, patho-

122 anatomical theories of spinal pain have so far failed to explain ongoing pain, or lead to reliable

123 treatments (Waddell 2004; O’Sullivan 2012). Brain-based treatments, including interventions

124 employing illusions, have been suggested as a way to directly target cortical representations 
125 (Senkowski and Heinz 2016; Moseley \& Flor 2012; Moseley, Gallace \& Spence 2012; Wand et 126 al., 2011; Stanton et al. 2016; Wallwork et al. 2015). It is suggested that illusory interventions, 127 such as mirror therapy, might assist by altering the way the painful body part is encoded in the 128 brain (Ramachandran \& Altschuler 2009). Because of the practical constraints of mirror 129 therapy — which relies on an unaffected and duplicated body part such as a hand or foot - it 130 cannot be used for spinal pain. Creating a perception of a healthy and free moving spine, 131 however, might be achieved using virtual reality.

132

133 Recent developments in virtual reality technology suggest that it can be used to alter perception 134 of the body in space. A virtual reality concept known as 're-directed walking' uses altered visual 135 feedback to manipulate perceived orientation, by shifting the virtual-world in ways that are not 136 aligned with real-world movement (Steinicke et al. 2010). The goal of this is to induce 137 corrections in real-world movement that, for example, enable a user to walk in a continuous 138 straight line in a virtual world, while remaining within constraints of real-world environments139 where they are walking in a circle (Steinicke et al. 2010). Preliminary tests suggest that 140 participants can be made to walk in a circle with a diameter as small as 22 metres, while being 141 convinced they are walking in a straight line. While re-directed walking pertains to whole body 142 movement through space, researchers have also developed ways of augmenting visual feedback 143 relating to motion of individual body segments within the body (Azmandian et al. 2016;

144 Kokkinara et al. 2016; Harvie et al. 2015; Harvie et al. 2016). For example, Azmandian et al.

145 (2016) showed that manipulating virtual arm movement can induce corresponding corrections in 146 actual limb movement. Such examples of interactions between visual input and motion, that 147 highlight the relationship between vision and bodily perception/kinesthesia, can be traced back to 148 von Helmholtz (1910), who's subjects mis-pointed at targets when wearing prism glasses that 149 displaced the visual field to one side. Our own research provides some evidence that such 150 illusory virtual movement may modulate chronic pain, by demonstrating that altered virtual neck 151 motion changes the relationship between real-world movement and pain, in people with 152 persistent neck pain (Harvie et al. 2015). While this is likely due to the capacity of vision to alter 153 perceived motion of the neck, the relationship between visual feedback and perceived neck 154 motion has not been investigated. Understanding the relationship between visual feedback and 
155 perceived neck motion (or other spine motion) may be important for developing therapeutic

156 applications for chronic pain based on altered visual-kinesthetic feedback.

157

158 In this study we aimed to establish whether altered visual-kinaesthetic feedback could change

159 perceived neck movement. We hypothesised that perceived neck movement would be dependent 160 on altered visual-kinaesthetic feedback.

161

162 Methods

163 Subjects

164 Twenty-four participants (14 males, age $(\mathrm{SD})=29(8))$, were recruited through flyers placed 165 around the University Campus. Participants were informed that we were testing how virtual166 reality affects body perception, but they were not informed that the visual feedback would be 167 systematically altered. Participants were excluded if they had current pain or history of chronic 168 pain. Based on a consistent effect seen in piloting, we expected a large effect size for the main

169 effect $\left(\left(\eta_{p}^{2}\right)=0.14\right)$. Based on the conventional $80 \%$ power, we estimated a sample of 10 people

170 was required. To improve sensitivity to detect the effect of additional variables such as

171 augmented auditory feedback, this sample was increased a priori to 24. All participants gave

172 written informed consent. The protocol was approved by the Institutional Ethics Committee of

173 Griffith University (Protocol Number: 2016/378).

174

\section{Equipment and software}

176 A virtual-reality (VR) technique known as redirected walking modulates visual-kinaesthetic

177 feedback by tracking real-world movement and then feeding this back into the virtual

178 environment in an understated or overstated form. This illusion is typically applied to the whole 179 body moving through space - to enable a user to walk continuously in a virtual world, while in 180 the spatial restraints of an indoor environment. We have previously modified this illusion to be 181 applied to individual joints or body segments relative to the rest of the body (Harvie et al. 2015; 182 Harvie et al. 2016.). We call this the Motor-Offset Visual Illusion (MoOVi) because the user real 183 world motor command is offset by given factor and then translated to a virtual world that can 184 under or overstate true movement. The factor by which real-world movement is translated to 185 virtual movement is known as the gain value. We used a VR head-mounted display (HMD) 
186 designed for immersive VR environments (Oculus Rift DK2; Oculus VR, Irvine, CA). Custom

187 built MoOVi software was used to map six scenes (captured with $360^{\circ}$ photography) to an

188 immersive virtual reality environment with three degrees of freedom (DOF), and to apply the

189 selected gain values (to visualize the MoOVi illusion see https://youtu.be/2Lu7G0cge2Q).

190

191 Measurement of kinesthetic drift

192 Participants were positioned in a well-supported chair that restricted torso movement.

193 Participants were asked to rotate only their head to a specific marker placed within the virtual

194 world, to stop, and to point in the direction that they felt they were facing (see Fig. 1). The digital

195 marker in the virtual world changed position depending on the gain value applied, such that it

196 always corresponded to $50^{\circ}$ degrees of real-world movement. A digital camera placed directly

197 above the participant, captured the participant's response. Markers were placed on the axis of

198 shoulder rotation, and the base of the index finger. The direction of perceived head rotation in

199 each trial was then extracted from screen shots using Kinovea video analysis software, by

200 placing digital markers in the location of the physical makers. Kinovea has previously shown to

201 be highly reliable for angular measures (Balsalobre-Fernández et al., 2014).

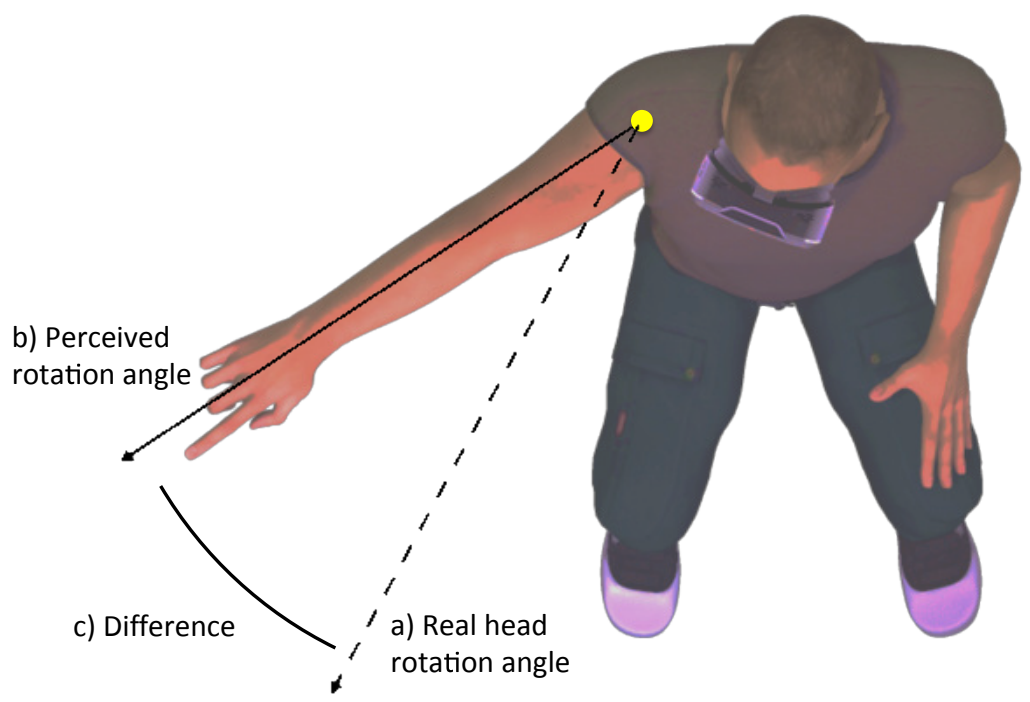

202

203 Fig. 1. Change in perceived body position 'c' was operationalized as the difference between

204 perceived head rotation ' $b$ ' after movement during altered visual feedback, relative to during

205 movement with normal visual feedback 'a'. 
207 Multisensory feedback

208 The principle manipulation was visual-feedback that under or overstated the amount of rotation

209 being performed by the participant. During neck movements to $50^{\circ}$ of rotation, the virtual reality 210 system administered corresponding visual-kinaesthetic feedback that was offset by a factor of $21150 \%$ to $200 \%$ (simulating between half and double the amount of movement actually occurring).

212 Real-world rotation to $50^{\circ}$ was kept constant to enabled relative control of proprioceptive and 213 vestibular inputs, such that the independent effects of the MoOVi illusion could be examined. To 214 test whether various additional multisensory and contextual factors influenced the strength of the 215 kinesthetic illusion, we divided the study into two parts.

217 Part one contained three multisensory conditions: 1. Vision only, 2. Vision + Sound, and, 3. 218 Vision + Sound + Avatar. In the Vision only condition, only visual feedback was provided. In 219 the Vision + sound condition, synchronous spatially defined auditory feedback was also 220 provided. The auditory feedback had spatial properties through manipulation of left-right ear 221 synchronicity and volume, which increased or decreased, relative to defined coordinates in the 222 virtual world. In the Vision + Sound + Avatar condition, a virtual body was included, to act as a 223 virtual anchor for the real body, such that the altered virtual head movement would be presented 224 as a relative to (a visual representation) of the rest of their body.

226 Part two of the study contained three contextual conditions designed to alter scene 227 familiarity/sense of immersion, and the $360^{\circ}$ photography based immersive virtual reality vs. a 228 modelled three-dimensional scene. These conditions were the: 1. Familiar Scene, constructed 229 from a $360^{\circ}$ photograph of the lab in which the experiment took place, 2 . Unfamiliar Scene $\left(360^{\circ}\right.$ 230 scene), and 3. Three-dimensional Scene (the same office scene in 3D). The $360^{\circ}$ immersive 231 virtual realities allow interactive viewing of panoramic photos in a spherical view. The three232 dimensional office scene provided a more complete virtual representation by imparting 233 dimensional and locational information for objects within the scene (i.e. table and chairs) 234 allowing stereo perception and improved perception of depth relative to the $360^{\circ}$ photographic 235 scenes. 


\section{Experimental design}

238 We used a within-subjects, repeated-measures design. In Part one, four gain settings were used:

$23950 \%, 100 \%, 150 \%$ and $200 \%$. All trials within each gain setting were undertaken in a single

240 block so as to minimize the likelihood of participants realizing our manipulation, and to

241 minimize the risk of motion sickness. The order of conditions was counterbalanced between

242 participants. Within each block, three sensory conditions were presented in randomized order.

243 These conditions were: 1. Vision only, 2. Vision + Sound, and, 3. Vision + Sound + Avatar.

244 Each condition was presented twice within each block, so that each block contained six trials.

245 This resulted in a total of 24 trials (i.e. four gain settings $\mathrm{x}$ three multisensory conditions $\mathrm{x}$ two

246 repetitions). In Part two, two different gain values were used: $100 \%$ and $180 \%$ to reduce the time

247 burden. All trials at each gain value were undertaken in a single block. The order of blocks, was

248 counterbalanced between participants. Within each block, three contextual conditions were

249 presented in random order. These conditions were: 1. Familiar Scene, 2. Unfamiliar Scene, 3.

250 Three-dimensional Scene. These conditions enabled us to determine whether familiarity and

251 dimensional cues could alter the strength of illusion and thus the primary outcome of perceived

252 movement. Each condition was presented twice within each block, so that each block contained

253 six trials. This resulted in a total of 12 trials (i.e. two settings $\mathrm{x}$ three contextual conditions $\mathrm{x}$ two

254 repetitions).

255

256 Manipulation checks

257 During the experiment, participants were asked to rotate their head to the right, to stop, and to

258 then physically point in the direction of perceived head orientation. We suspected that the real-

259 time shift in perceived head orientation during rotation might be diluted through the post-

260 movement task of actively considering their orientation, because internal reference frames, based

261 on proprioceptive and vestibular information, might have greater time to compete with the bogus

262 visual information. Thus, at the end of the experiment, participants were asked to turn their head

263 to the minimum and maximum real-world movement that they perceived across the trials. By

264 comparing these head rotation angles to actual head movement (always $50^{\circ}$ ), and to the pointing

265 data, we aimed to gauge potential bias in the pointing data, and obtain a secondary measure of

266 the overall magnitude of kinaesthetic drift. 


\section{Statistical treatment}

269 In order to test the main hypothesis that visual information that overstates or understates true 270 rotation alters perceived movement, we compared perceived head orientation between visual

271 feedback conditions, using repeated-measures ANOVA with Bonferroni-corrected pairwise 272 comparisons. To test for interactions with multisensory and contextual/dimensional variables, 273 these factors were entered as covariates. Prior to analysis, perceived orientation in each condition 274 was converted to a difference score in degrees, relative to their average perceived head 275 orientation across conditions. Alpha was set at $p=0.05$. The magnitude of the effects were 276 quantified using partial eta squared $\left(\eta_{p}^{2}\right)$ and interpreted with respect to Cohen's guidelines $(0.01$ $277=$ small, $0.059=$ medium and $0.138=$ large $($ Cohen, 1988). Based on the hypothesis that 278 individual factors might influence the effect of conflicting sensory information on perceived 279 neck movement, we also conducted exploratory analyses to determine if the effect related to 280 gender or simulator sickness susceptibility. To do this we calculated the slope coefficient created 281 by the increasing perceived head orientation angles, at the four increasing rotation gain settings, 282 for each participant. To test whether the effect related to gender we performed an Independent 283 Samples T-test comparing the slope coefficient in males vs. females. To test whether the effect 284 related to susceptibility to motion sickness, we performed a Pearson's correlation analysis 285 between simulator sickness score and the slope coefficient representing the effect.

287 Results

288 Participants

289 Forteen males and 10 females participated. Average age of participants was 30 years (range: 20290 42). Following the experiment, participants reported only mild motion sickness symptoms: mean 291 (range) Simulator Sickness Questionnaire = 8\% (0-38\%). Only one participant scored over 20\%, 292 and most participants reporting a very mild sense of nausea.

293

294 Kinaesthetic drift

295 A linear relationship was observed between change in visual-feedback and change in perceived 296 movement (see Figure 2). The overall effect of altered visual feedback was $7.5(6.4)^{\circ}$ as measured 297 using the pointing task. Visual feedback that overstated real-world movement by a factor of 2 , 
298 resulted in $4.6(5)^{\circ}$ drift in perceived orientation, whereas understating real-wold movement by a 299 factor of .5 , resulted in on average $-2.9(3.3)^{\circ}$ drift in perceived orientation (see Table 1 for results 300 by condition and gain factor). The repeated measures ANOVA for Part 1 confirmed a large 301 effect of altered sensory feedback $\left(F(3,92)=6.4, p=0.001, \eta_{p}^{2}=0.17\right)$ on perception of head 302 movement (Fig. 2). That is, altered visual feedback caused a proprioceptive drift in the direction 303 of the visually suggested movement. The magnitude of the drift was not moderated by the 304 addition of illusory auditory feedback, nor by the presence of the avatar $(F(3,92)=1.5, p=0.2$, $\left.305 \eta_{p}^{2}=0.05\right)$. The repeated measures ANOVA for Part 2 also revealed a large effect of altered 306 sensory feedback $\left(F(1,94)=16.5, p<0.001, \eta_{p}^{2}=0.15\right)$ on perception of head movement, 307 confirming the findings of Part 1 . The magnitude of the drift was not moderated by scene 308 familiarity or three-dimensionality $\left(F(1,94)=0.2, p=0.7, \eta_{p}^{2}<0.01\right)$.

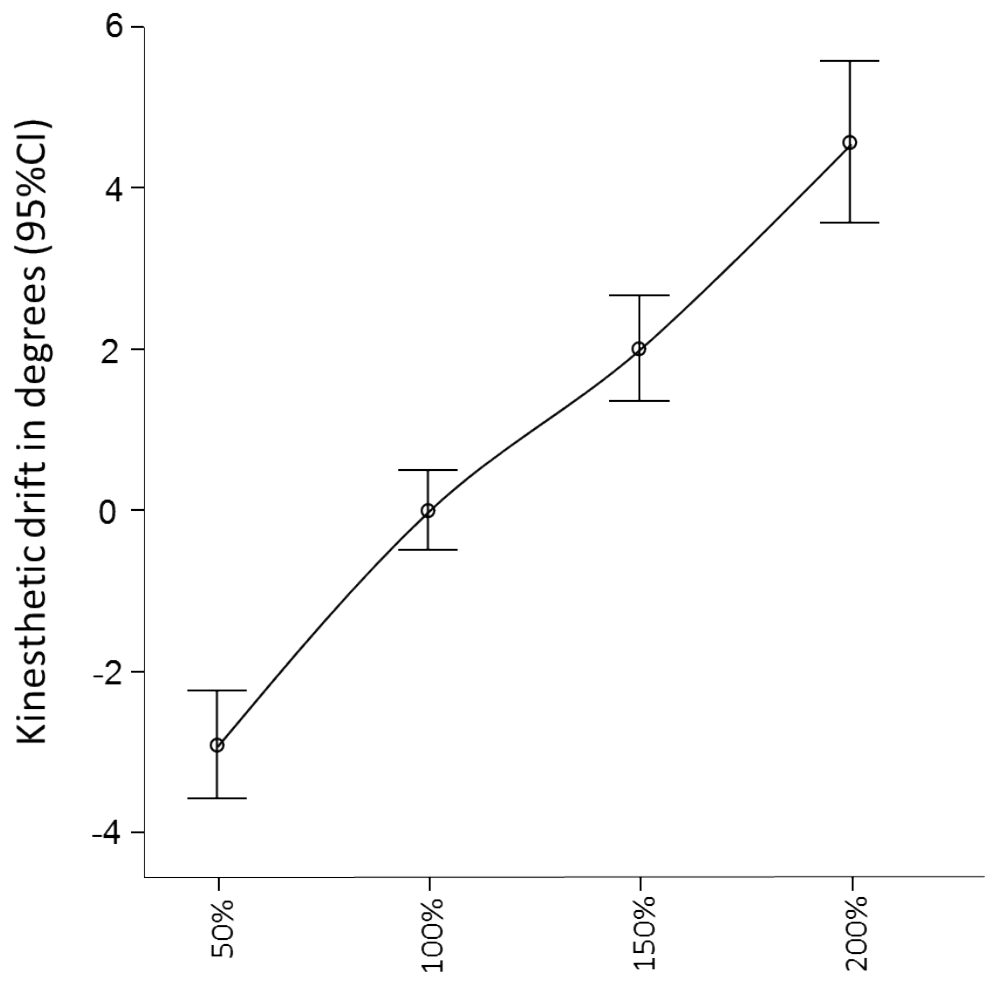
Virtual rotation as a percentage of real rotation

Fig. 2. Perceived direction of head orientation, relative to average perceived direction across conditions. 


\begin{tabular}{lllll}
\hline & \multicolumn{5}{c}{ Gain factor } \\
\hline Sensory condition - Part 1 & $\mathbf{0 . 5}$ & $\mathbf{1}$ & $\mathbf{1 . 5}$ & $\mathbf{2}$ \\
\hline Vision & $-1.8(3.7)$ & $1.4(2.4)$ & $2.1(2.6)$ & $5.1(4.8)$ \\
Vision+Avatar & $-3.8(3.0)$ & $-1.0(1.9)$ & $1.5(2.0)$ & $4.2(4.0)$ \\
Vision+Avatar+Audio & $-2.9(3.1)$ & $-0.5(2.7)$ & $1.8(3.3)$ & $4.0(5.6)$ \\
Vision+Audio & $-3.2(3.2)$ & $0.0(2.1)$ & $2.7(4.6)$ & $5.0(5.4)$ \\
Overall & $-2.9(3.3)$ & $0.0(2.4)$ & $2.0(3.2)$ & $4.6(5.0)$ \\
\hline Sensory condition - Part 2 & $\mathbf{1}$ & $\mathbf{1 . 8}$ & & \\
\hline 3D & $0.2(1.6)$ & $3.6(3.6)$ & & \\
Familiar & $-0.2(1.8)$ & $3.6(4.4)$ & & \\
Unfamiliar & $0.2(1.5)$ & $3.9(4.3)$ & & \\
Overall & $0.0(1.6)$ & $3.5(3.8)$ & & \\
\hline
\end{tabular}

316

317 Table 1. Change in perceived movement [Mean(SD)] for each gain factor and sensory condition 318 as measured by the pointing task.

Manipulation check: perceived head orientation

321 When asked to reproduce the range of head orientation angles performed while in the virtual

322 world, participants on average perceived between $31^{\circ}(\mathrm{SD} 7$, range $21-45)$ and $65^{\circ}(\mathrm{SD} 13$, range

323 47-97), despite always turning to $50^{\circ}$. Paired t-test confirmed the statistical difference between

324 the smallest and the largest perceived head rotation $\left(p<0.001\right.$; mean difference $=33^{\circ}[\mathrm{SD} 12$, 325 range 18-70]).

326

327 Exploratory analysis

328 The slope coefficient representing the effect also did not differ by gender ( $\mathrm{t}(22)-1.7, p=.105)$,

329 although a trend towards a greater effect in females was noted [Slope coefficient; Males =

$3301.9(1.3)$, Females $=3.2(2.3)]$. No correlation was shown between simulator sickness scores and 331 tendency to experience greater kinaesthetic $\operatorname{drift}(p=.6, \mathrm{r}=-.11)$.

\section{Discussion}


334 We aimed to establish whether altered kinaesthetic feedback could change perceived neck

335 movement. We hypothesised that perceived neck movement would be dependent on altered

336 visual-kinaesthetic feedback. Our hypothesis was confirmed, as evidenced by main effect of gain

337 value on perceived orientation and that perceived orientation followed gain value in a systematic

338 way. The effectiveness of the illusion seems to be unaffected by the addition of cross-modal

339 (auditory) input, nor by scene familiarity. While the kinaesthetic drift demonstrated by the

340 pointing task was modest, the manipulation check suggested that this action captured only a

341 portion of the perceptual shift. This is analogous to the drift in perceived location of the hand

342 during the rubber hand illusion, where asking participants with closed eyes to point to where they

343 feel their hand to be, captures only a portion of the real-time perceptual shift (Botvinick \& Cohen

344 1998). The current findings demonstrate that virtual reality can induce illusory neck movement

345 analogous to the body-illusion underlying mirror therapy for pathological and phantom limb

346 pain. The finding is consistent with other virtual reality studies showing that altered visual

347 feedback can manipulate whole body (Steinicke et al., 2015) and limb (Kokkinara et al. 2015;

348 Azmandian et al. 2016) movement.

349

350 The Motor Offset Visual Illusion (MoOVi)

351 Perceptual models explain visual illusions by the way that perceptions are derived from

352 inferences about the world, derived from past experience and multisensory information that is

353 uncertain and sometimes conflicting (Cheng et al. 2007). In the current example, perception of

354 body orientation would be informed most notably by: 1. Expected movement, 'the efferent copy

355 of the motor command', 2. Proprioceptive and vestibular feedback, and 3. The altered visual-

356 kinaesthetic feedback. Thus, the resulting perception of head orientation is a best guess inference

357 based on the relative influence of these variables. As demonstrated by other bodily illusions,

358 visual information is weighted heavily and therefore shows a particular propensity to influence

359 perception. Perceptual models explain this by suggesting that the most reliable sensory channel is

360 weighted most heavily and, with respect to spatial data, vision is the most reliable sensory

361 channel (Cheng et al. 2007). This idea is also consistent with the principle that the influence of

362 one neural network on another depends in part on its precision (see cite for relevant reviews

363 Nicolelis \& Lebedev 2009; Wallwork et al. 2015; Wallwork et al. in press). 
365 While the magnitude of change in perceived head rotation, as measured by the primary outcome,

366 does not appear consistent with the heavy weighting of visual information, our manipulation

367 check suggests that the actual manipulation of perceived movement was much greater. A

368 different methodology may be more capable of objectifying the change in perceived head

369 orientation (see Limitations and future directions). Nonetheless, the magnitude of the effect is

370 consistent with similar measures of kinaesthetic drift previously used, and which suggest such

371 measures capture only a portion of the change in body position experienced during the illusion.

372 For example, despite the convincingness of the rubber hand illusion, participants show a

373 kinaesthetic drift of only $23 \mathrm{~mm}$ towards the rubber hand, when measured by a pointing task

374 (Botvinick \& Cohen 1998). In contrast, observing real-time changes movement behaviour in

375 response to altered feedback may be more a more sensitive measure. For example, Kokkinara et

376 al. (2016) observed up to $22^{\circ}$ of drift in arm movement in response to modulation of virtual arm

377 movement.

378

379

380

Illusory movement as therapy? Plausible mechanisms

381 To date, persistent pain treatments targeting suspected tissue pathology have shown to have very

382 limited or no ongoing benefit (see e.g. van Tulder et al., 2006a; van Tulder et al., 2006b). The

383 quest for better treatments has seen attention shift towards targeting central mechanisms (Wand

384 et al. 2011; Moseley \& Flor 2012). While cognitive and behavioural approaches targeting

385 thoughts, beliefs and behaviors undoubtedly have potential to alter neural processes associated

386 with pain, considering brain-based approaches from a perceptual and brain science perspective

387 will likely lead to a range of different treatment avenues. Recent perspectives highlight several

388 pathways by which perception-altering associative learning processes might lead to chronic pain

389 (Moseley \& Vlaeyen 2015; Tabor et al. in publication; Zaman et al. 2015). Further recent

390 reviews have highlighted a potential role for multisensory illusions in developing future

391 treatment opportunities (Senkowski and Heinz 2016; Moseley \& Flor 2014; Boesch et al. 2016).

392 It follows that similar mechanisms applied differently might reverse some of these processes.

393

394 The view of chronic pain employed here, is one where pain persists because the affected body

395 part(s) in someway continue to be represented in the brain as being under threat and requiring 
396 certain protections - perhaps because the injurious event-related encoding persists (Moseley \&

397 Vlaeyen 2015). One way to consider altering this threatening body-related neural encoding is to

398 disconfirm implicit expectations of pain, such as those associated with movements. This requires

399 an experience that is normally painful to be experienced without pain. Such violations of

400 expectation are known to be powerful drivers of learning (Rescorla \& Wagner, 1972), and are

401 thought to alter prior implicit expectancies that may drive the resulting pain responses (Tabor et

402 al. in press).

403

404 Under normal clinical conditions, creating pain-free or non-threatening movement experiences,

405 through which to extinguish implicit expectations of pain, can be difficult. The MoOVi illusion

406 presents an opportunity for users to experience ranges of pain-free movement that exceed the

407 expected range of pain-free movement. Doing so may assist to reinstate a less threatened bodily

408 representation. Indeed we have created a Samsung Galaxy Gear application that patients can use

409 at home that aims to serve this aim. The app instructs a rotation exercises and progressively

410 exaggerates these movements over a number of repetitions, while limiting real-world movement

411 to within pain-free limits through a calibration process. We are shortly commencing initial

412 clinical testing of this application for the treatment of chronic neck pain, where central

413 mechanisms are thought to play a role (See Australia and New Zealand Trial Registry, Universal

414 Trial Number: U1111-1190-8259). This is conceptually similar, not only to mirror therapy, but to

415 Graded Motor Imagery (GMI) - which targets progressive activation of motor processes

416 without triggering unwanted protective responses (Moseley 2004; Moseley 2006). Indeed the

417 final phase of three in graded motor imagery is illusory movement with mirror therapy, which

418 has preliminary evidence of benefit for arm pain (Bowering et al. 2013). The current results raise

419 the possibility that the MoOVi illusion might allow a graded motor imagery-like approach to

420 neck and other spinal pain conditions.

421

422 Limitations and future directions

423 As mentioned, the change in perceived movement due to the bogus visual feedback was

424 considerably less than we expected. However, our manipulation check suggested the primary

425 outcome was diluted and further research should explore better ways to assess the magnitude of

426 the illusion and its effect on motor output. For example, we suspect that in the process of 
427 reaching out, the illusion may have been partially broken because the expectation of seeing the

428 arm in the visual field would have been violated. The use of a virtual arm, or a reaching task

429 below the visual field may be a more appropriate measure. While auditory and contextual cues

430 did not appear to modulate the strength of the illusion in this study, it is possible that our

431 measure was simply too insensitive to detect its effect. Supporting this thesis, is a recent study

432 showing that, that in general, a greater number of additional sensory cues - including

433 vibrotactile, wind and sound - corresponds to improved performance and immersiveness in

434 virtual environments (Feng et al. 2016).

435

436 While the current study is framed with respect to chronic pain, its assessment and treatment, this

437 is clearly not a clinical study. The potential benefit is speculative and further research is required

438 before taking this to a clinical phase. A further direction for future study, is examining individual

439 factors that relate to susceptibility to this kinaesthetic illusion. For example, sensitivity to

440 interoceptive information appears to modulate the degree of kinaesthetic drift induced by the

441 rubber hand illusion (Tsakiris et al. 2011). In the therapeutic context, identifying factors that

442 predict sensitivity to the illusion may be relevant in predicting treatment efficacy. While gender

443 and susceptibility to simulator sickness did not significantly predict effectiveness of the illusion,

444 future research might investigate whether other individual factors modulate the effect.

445

446 Conclusion

447 Virtual reality can be used to augment perceived movement and body position, such that one can

448 perform small real-world movements, yet experience a larger movement. The current study

449 extends this finding to movement of the neck. Thus, the MoOVi technique may have similar

450 central effects and therapeutic potential to treatments currently only applied to the limbs, for

451 example mirror therapy.

452

453

454

455

456

457 
459

460

461

462

463

464

465

466

467

468

469

470

471

472

473

474

475

476

477

478

479

480

481

482

483

484

485

486

487

488

489

490

491

492

493

494

495

496

497

498

499

500

501

502

\section{References}

Azmandian, M., Hancock, M., Benko, H., Ofek, E., \& Wilson, A. D. (2016, May). Haptic Retargeting Video Showcase: Dynamic Repurposing of Passive Haptics for Enhanced Virtual Reality Experience. In Proceedings of the 2016 CHI Conference Extended Abstracts on Human Factors in Computing Systems (pp. 3-3). ACM.

Balsalobre-Fernández, C., Tejero-González, C. M., del Campo-Vecino, J., \& Bavaresco, N. (2014). The concurrent validity and reliability of a low-cost, high-speed camera-based method for measuring the flight time of vertical jumps. The Journal of Strength \& Conditioning Research, 28(2), 528-533.

Barnsley, N., McAuley, J. H., Mohan, R., Dey, A., Thomas, P., \& Moseley, G. L. (2011). The rubber hand illusion increases histamine reactivity in the real arm. Current Biology, 21(23), R945-R946.

Boesch, E., Bellan, V., Moseley, G. L., \& Stanton, T. R. (2016). The effect of bodily illusions on clinical pain: a systematic review and meta-analysis. Pain,157(3), 516-529.

Botvinick, M., \& Cohen, J. (1998). Rubber hands' feel'touch that eyes see. Nature, 391(6669), 756-756.

Bowering, K. J., O'Connell, N. E., Tabor, A., Catley, M. J., Leake, H. B., Moseley, G. L., \& Stanton, T. R. (2013). The effects of graded motor imagery and its components on chronic pain: a systematic review and meta-analysis.The Journal of Pain, 14(1), 3-13.

Cacchio, A., De Blasis, E., De Blasis, V., Santilli, V., \& Spacca, G. (2009). Mirror therapy in complex regional pain syndrome type 1 of the upper limb in stroke patients. Neurorehabilitation and neural repair.

Catley, M. J., O'Connell, N. E., Berryman, C., Ayhan, F. F., \& Moseley, G. L. (2014). Is tactile acuity altered in people with chronic pain? A systematic review and meta-analysis. The Journal of Pain, 15(10), 985-1000.

Chan, B. L., Witt, R., Charrow, A. P., Magee, A., Howard, R., Pasquina, P. F., ... \& Tsao, J. W. (2007). Mirror therapy for phantom limb pain. New England Journal of Medicine, 357(21), 2206-2207.

Cheng, K., Shettleworth, S. J., Huttenlocher, J., \& Rieser, J. J. (2007). Bayesian integration of spatial information. Psychological bulletin, 133(4), 625.

Di Pietro, F., McAuley, J. H., Parkitny, L., Lotze, M., Wand, B. M., Moseley, G. L., \& Stanton, T. R. (2013). Primary somatosensory cortex function in complex regional pain syndrome: a systematic review and meta-analysis.The Journal of Pain, 14(10), 1001-1018. 
503 Di Pietro, F., Stanton, T. R., Moseley, G. L., Lotze, M., \& McAuley, J. H. (2015).

504 Interhemispheric somatosensory differences in chronic pain reflect abnormality of the healthy

505 side. Human brain mapping, 36(2), 508-518.

506

507

508

509

510

511

512

513

514

515

516

517

518

519

520

521

522

523

524

525

526

527

528

529

530

531

532

533

534

535

536

537

538

539

540

541

542

543

544

545

546

547

548

Feng, M., Arindam D., \& Lindeman. R. (2016). An initial exploration of a multi-sensory design space: Tactile support for walking in immersive virtual environments. 3D User Interfaces, IEEE Symposium, 95-104.

Flor, H., Elbert, T., Knecht, S., Wienbruch, C., Pantev, C., Birbaumer, N., ... \& Taub, E. (1995). Phantom-limb pain as a perceptual correlate of cortical reorganization following arm amputation. Nature, 375(6531), 482-484.

Flor, H., Braun, C., Elbert, T., \& Birbaumer, N. (1997). Extensive reorganization of primary somatosensory cortex in chronic back pain patients. Neuroscience letters, 224(1), 5-8.

Harvie, D. S., Broecker, M., Smith, R. T., Meulders, A., Madden, V. J., \& Moseley, G. L. (2015). Bogus visual feedback alters onset of movement-evoked pain in people with neck pain. Psychological science, 0956797614563339.

Harvie, D. S., Hillier, S., Madden, V. J., Smith, R. T., Broecker, M., Meulders, A., \& Moseley, G. L. (2016). Neck pain and proprioception revisited using the proprioception incongruence detection test. Physical Therapy, 96(5), 671-678.

Juottonen, K., Gockel, M., Silén, T., Hurri, H., Hari, R., \& Forss, N. (2002). Altered central sensorimotor processing in patients with complex regional pain syndrome. Pain, 98(3), 315-323.

Kokkinara, E., Slater, M., \& López-Moliner, J. (2015). The effects of visuomotor calibration to the perceived space and body, through embodiment in immersive virtual reality. $A C M$ Transactions on Applied Perception (TAP), 13(1), 3.

Marinus, J., Moseley, G. L., Birklein, F., Baron, R., Maihöfner, C., Kingery, W. S., \& van Hilten, J. J. (2011). Clinical features and pathophysiology of complex regional pain syndrome. The Lancet Neurology, 10(7), 637-648.

Moseley, G. L. (2004). Graded motor imagery is effective for long-standing complex regional pain syndrome: a randomised controlled trial. Pain, 108(1), 192-198.

Moseley, G. L. (2006). Graded motor imagery for pathologic pain A randomized controlled trial. Neurology, 67(12), 2129-2134.

Moseley, G. L., \& Butler, D. S. (2015). Fifteen years of explaining pain: the past, present, and future. The Journal of Pain, 16(9), 807-813.

Moseley, G. L., \& Flor, H. (2012). Targeting cortical representations in the treatment of chronic pain a review. Neurorehabilitation and neural repair,26(6), 646-652. 
549 Moseley, G. L., Gallace, A., \& Spence, C. (2012). Bodily illusions in health and disease:

550 physiological and clinical perspectives and the concept of a cortical 'body matrix'. Neuroscience

551 \& Biobehavioral Reviews, 36(1), 34-46.

552

553

554

555

556

557

558

559

560

561

562

563

564

565

566

567

568

569

570

571

572

573

574

575

576

577

578

579

580

581

582

583

584

585

586

587

588

589

590

591

592

593 Vos, T., Barber, R. M., Bell, B., Bertozzi-Villa, A., Biryukov, S., Bolliger, I., ... \& Duan, L.

594 (2015). Global, regional, and national incidence, prevalence, and years lived with disability for 
595301 acute and chronic diseases and injuries in 188 countries, 1990-2013: a systematic analysis

596 for the Global Burden of Disease Study 2013. The Lancet, 386(9995), 743-800.

597

598

599

600 Wallwork, S. B., Bellan, V., Catley, M. J., \& Moseley, G. L. (2015). Neural representations and

601 the cortical body matrix: implications for sports medicine and future directions. British journal of

602 sports medicine, bjsports-2015.

603

604 Wallwork SB, Bellan V, GL M. Pain, bodily protection and the relevance of neural rehabilitation 605 after dance injury. Journal of Dance Medicine and Science In Press; In Press.

606

607

Wand, B. M., Parkitny, L., O’Connell, N. E., Luomajoki, H., McAuley, J. H., Thacker, M., \&

608 Moseley, G. L. (2011). Cortical changes in chronic low back pain: current state of the art and

609

610

611 Zaman, J., Vlaeyen, J. W., Van Oudenhove, L., Wiech, K., \& Van Diest, I. (2015). Associative

612

613

614

615

616

617 pain. Neuroscience \& Biobehavioral Reviews, 51, 118-125. 